\title{
Homogeneous balls in a spontaneously broken U(1) gauge theory
}

\author{
Hideki Ishihara $^{*}$ and Tatsuya Ogawa ${ }^{\dagger}$ \\ Department of Mathematics and Physics, Graduate School of Science, \\ and Nambu Yoichiro Institute of Theoretical and Experimental Physics (NITEP), \\ Osaka City University, Osaka 558-8585, Japan
}

(Received 29 January 2019; published 29 March 2019)

\begin{abstract}
We study the coupled system consisting of a complex matter scalar field, a U(1) gauge field, and a complex Higgs scalar field that causes spontaneously symmetry breaking. We show by numerical calculations that there are spherically symmetric nontopological soliton solutions. Homogeneous balls solutions, all fields take constant values inside the ball and in the vacuum state outside, appear in this system. It is shown that the homogeneous balls have the following properties: the charge density of the matter scalar field is screened by a counter charge cloud of the Higgs and gauge field everywhere; an arbitrarily large size is allowed; the energy density and pressure of the ball behave as a homogeneous nonrelativistic gas; a large ball is stable against dispersion into free particles and against decay into two smaller balls.
\end{abstract}

DOI: $10.1103 /$ PhysRevD.99.056019

\section{INTRODUCTION}

A class of interesting excitations in field theories is solitons, i.e., nonlinear solutions localized in finite spatial regions. The solitons are classified into two types: topological and nontopological solitons. The former are field configurations with a topological charge that is invariant under continuous deformations of the field with fixed boundary conditions. The topological solitons cannot relax to zero energy configurations due to conserved topological quantities. The latter represent field configurations with the lowest energy for a fixed conserved charge in global U(1)-invariant theories, where the symmetry of the systems guarantee the stability. Friedberg et al. [1] introduced nontopological solitons in a coupled system of a complex scalar field and a self-interacting real scalar field. Coleman [2] showed the simplest example of nontopological solitons, so-called Q balls, ${ }^{1}$ can appear in a system of a self-interacting single complex scalar field.

The Q balls attract much attention because the Q balls generally appear in theories with potentials inspired by supersymmetric theories that include global U(1) symmetries [3-5]. Furthermore, in a cosmological context, the Q ball is a candidate of the dark matter of the Universe [6-10] and a source for baryogenesis [11-13].

\footnotetext{
*ishihara@sci.osaka-cu.ac.jp

†taogawa@sci.osaka-cu.ac.jp

${ }^{1}$ Hereafter, we call a spherically symmetric nontopological soliton a $\mathrm{Q}$ ball, in short.

Published by the American Physical Society under the terms of the Creative Commons Attribution 4.0 International license. Further distribution of this work must maintain attribution to the author(s) and the published article's title, journal citation, and DOI. Funded by SCOAP.
}

Generalizations of the $\mathrm{Q}$ balls in local U(1)-invariant theories by introduction of a gauge field are also studied [14-18]. There are significant differences between gauged and ungauged $\mathrm{Q}$ balls. For example, an ungauged $\mathrm{Q}$ ball with an arbitrarily large charge is allowed while an upper bound of the charge appears for a gauged Q ball [14-16]. Otherwise, a complicated form of the potential should be assumed for the existence of large Q balls $[17,18]$.

In this paper, we consider a gauge theory with spontaneous symmetry breaking, which is a fundamental framework of modern physics. We study the system consisting of a complex scalar field as matter, a U(1) gauge field, and a complex Higgs scalar field that causes spontaneous symmetry breaking: a local $\mathrm{U}(1) \times$ global $\mathrm{U}(1)$ symmetry breaks to a global U(1) symmetry. While models with a single scalar field are assumed to have complicated selfinteractions, e.g., third or sixth order potentials, or nonpolynomial potentials, for the existence of $Q$ balls, we show the existence of $\mathrm{Q}$ balls in the model that has simple natural interaction terms. Then, this work would suggest $Q$ balls can appear in a wide class of gauge theories.

We assume stationary and spherically symmetric configurations of the fields, and reduce the system into a coupled ordinary differential equations. We show Q balls exist in this theory by using a numerical method. ${ }^{2}$ The all fields are nonvanishing in a finite region while the matter scalar field and the gauge field vanish, and the Higgs field takes the vacuum expectation value outside the region. Phase rotation of the complex Higgs scalar field is absorbed by the gauge field, and phase rotation of the complex matter

\footnotetext{
${ }^{2}$ This was reported briefly in Ref. [19].
} 
scalar field, which represents charge, characterizes the solutions. There are two types of solutions classified by the shape: Gaussian balls, expressed by the Gaussian-like functions, and homogeneous balls, expressed by steplike functions. In this paper, we concentrate on the homogeneous balls, which are described by bounce solutions that connect two stationary points of the ordinary differential equations and clarify their properties.

We show that the homogeneous balls in the present system have the following properties. The charge density of the matter scalar field of a homogeneous ball is screened everywhere by a counter charge cloud of the Higgs and gauge fields, namely, perfect screening occurs [20]. A homogeneous ball has constant energy density and pressure inside the ball, and the pressure is much smaller than the energy density; i.e., the homogeneous ball is like a ball of nonrelativistic gas. A homogeneous ball with an arbitrarily large size is allowed in contrast to a gauged Q ball without a Higgs field has an upper limit of size. A large homogeneous ball is stable against dispersion into free particles and decays into two smaller $\mathrm{Q}$ balls.

The paper is organized as follows. In Sec. II, we present the basic model investigated in this paper and show that the model is described by a coupled system of ordinary differential equations. In Sec. III, we obtain numerical solutions that represent Q balls, and we see charge screening. We analyze properties of the homogeneous ball solutions in Sec. IV and the stability of Q balls in the present system in Sec. V. Section VI is devoted to a summary and discussions.

\section{BASIC MODEL}

We consider the action given by

$$
\begin{aligned}
S= & \int d^{4} x\left(-\left(D_{\mu} \psi\right)^{*}\left(D^{\mu} \psi\right)-\left(D_{\mu} \phi\right)^{*}\left(D^{\mu} \phi\right)\right. \\
& \left.-V(\phi)-\mu \psi^{*} \psi \phi^{*} \phi-\frac{1}{4} F_{\mu \nu} F^{\mu \nu}\right),
\end{aligned}
$$

where $\psi$ is a complex matter scalar field, $\phi$ is a complex Higgs scalar field with the potential,

$$
V(\phi):=\frac{\lambda}{4}\left(\phi^{*} \phi-\eta^{2}\right)^{2},
$$

where $\lambda$ and $\eta$ are positive constants, and $F_{\mu \nu}:=\partial_{\mu} A_{\nu}-$ $\partial_{\nu} A_{\mu}$ is the field strength of a $\mathrm{U}(1)$ gauge field $A_{\mu}$. The covariant derivative $D_{\mu}$ in (1) is defined by

$$
D_{\mu} \psi:=\partial_{\mu} \psi-i e A_{\mu} \psi, \quad D_{\mu} \phi:=\partial_{\mu} \phi-i e A_{\mu} \phi,
$$

where $e$ is a gauge coupling constant. This model is a generalization of the Friedberg-Lee-Sirlin model by introducing a complex Higgs scalar field and a U(1) gauge field.
The action (1) is invariant under the local U(1) times the global U(1) gauge transformations,

$$
\begin{gathered}
\psi(x) \rightarrow \psi^{\prime}(x)=e^{i(\chi(x)-\gamma)} \psi(x), \\
\phi(x) \rightarrow \phi^{\prime}(x)=e^{i(x(x)+\gamma)} \phi(x), \\
A_{\mu}(x) \rightarrow A_{\mu}^{\prime}(x)=A_{\mu}(x)+e^{-1} \partial_{\mu} \chi(x),
\end{gathered}
$$

where $\chi(x)$ and $\gamma$ are an arbitrary function and a constant, respectively. Owing to the gauge invariance, there are the conserved currents,

$$
\begin{aligned}
j_{\psi}^{\nu} & :=i e\left\{\psi^{*}\left(D^{\nu} \psi\right)-\psi\left(D^{\nu} \psi\right)^{*}\right\}, \\
j_{\phi}^{\nu} & :=i e\left\{\phi^{*}\left(D^{\nu} \phi\right)-\phi\left(D^{\nu} \phi\right)^{*}\right\},
\end{aligned}
$$

satisfying $\partial_{\mu} j_{\psi}^{\mu}=0$ and $\partial_{\mu} j_{\phi}^{\mu}=0$. Consequently, the total charge of $\psi$ and $\phi$ defined by

$$
\begin{aligned}
Q_{\psi} & :=\int \rho_{\psi} d^{3} x, \\
Q_{\phi} & :=\int \rho_{\phi} d^{3} x,
\end{aligned}
$$

are conserved, where $\rho_{\psi}:=j_{\psi}^{t}$ and $\rho_{\phi}:=j_{\phi}^{t}$.

The energy of the system is given by ${ }^{3}$

$$
\begin{aligned}
E= & \int d^{3} x\left(\left|D_{t} \psi\right|^{2}+\left(D_{i} \psi\right)^{*}\left(D^{i} \psi\right)+\left|D_{t} \phi\right|^{2}+\left(D_{i} \phi\right)^{*}\left(D^{i} \phi\right)\right. \\
& \left.+V(\phi)+\mu|\psi|^{2}|\phi|^{2}+\frac{1}{2}\left(E_{i} E^{i}+B_{i} B^{i}\right)\right)
\end{aligned}
$$

where $E_{i}:=F_{i 0}, \quad B^{i}:=1 / 2 \epsilon^{i j k} F_{j k}$, and $i$ denotes a spatial index. In the vacuum state, which minimizes the energy (11), the fields $\psi, \phi$, and $A_{\mu}$ should satisfy

$$
\psi=0, \quad \phi^{*} \phi=\eta^{2}, \quad D_{\mu} \phi=0, \quad \text { and } F_{\mu \nu}=0 ;
$$

equivalently,

$$
\psi=0, \quad \phi=\eta e^{i \theta(x)}, \quad \text { and } \quad A_{\mu}=e^{-1} \partial_{\mu} \theta,
$$

where $\theta$ is an arbitrary continuous regular function. We exclude topologically nontrivial case in this paper. The Higgs scalar field $\phi$ has the vacuum expectation value $\eta$, then the $\mathrm{U}_{\text {local }}(1) \times \mathrm{U}_{\text {global }}(1)$ symmetry is broken into a global U(1) symmetry, so that the gauge field $A_{\mu}$ and the complex scalar field $\psi$ acquire the mass $m_{A}=\sqrt{2} e \eta$ and $m_{\psi}=\sqrt{\mu} \eta$, respectively. The real scalar field that denotes a

\footnotetext{
${ }^{3}$ See the Appendix.
} 
fluctuation of the amplitude of $\phi$ around $\eta$ acquires the mass $m_{\phi}=\sqrt{\lambda} \eta$.

By varying (1) with respect to $\psi^{*}, \phi^{*}$, and $A_{\mu}$, we obtain the equations of motion,

$$
\begin{gathered}
D_{\mu} D^{\mu} \psi-\mu \phi^{*} \phi \psi=0 \\
D_{\mu} D^{\mu} \phi-\frac{\lambda}{2} \phi\left(\phi^{*} \phi-\eta^{2}\right)-\mu \phi \psi^{*} \psi=0, \\
\partial_{\mu} F^{\mu \nu}=j_{\phi}^{\nu}+j_{\psi}^{\nu} .
\end{gathered}
$$

We assume that the fields are stationary and spherically symmetric in the form,

$$
\begin{gathered}
\psi=e^{i \omega t} u(r), \\
\phi=e^{i \omega^{\prime} t} f(r), \\
A_{t}=A_{t}(r), \quad \text { and } \quad A_{i}=0,
\end{gathered}
$$

where $\omega$ and $\omega^{\prime}$ are constants, and $u(r)$ and $f(r)$ are real functions of $r$. Using the gauge transformation (4), (5), and (6), we fix the variables as

$$
\begin{gathered}
\phi(r) \rightarrow f(r), \\
\psi(t, r) \rightarrow e^{i \Omega t} u(r):=e^{i\left(\omega-\omega^{\prime}\right) t} u(r), \\
A_{t}(r) \rightarrow \alpha(r):=A_{t}(r)-e^{-1} \omega^{\prime},
\end{gathered}
$$

where we assume $\Omega:=\omega-\omega^{\prime}>0$ without the loss of generality.

Substituting (20), (21), and (22) into (14), (15), and (16), we obtain a set of the ordinary differential equations,

$$
\begin{gathered}
\frac{d^{2} u}{d r^{2}}+\frac{2}{r} \frac{d u}{d r}+(e \alpha-\Omega)^{2} u-\mu f^{2} u=0 \\
\frac{d^{2} f}{d r^{2}}+\frac{2}{r} \frac{d f}{d r}+e^{2} f \alpha^{2}-\frac{\lambda}{2} f\left(f^{2}-\eta^{2}\right)-\mu f u^{2}=0 \\
\frac{d^{2} \alpha}{d r^{2}}+\frac{2}{r} \frac{d \alpha}{d r}+\rho_{\text {total }}=0
\end{gathered}
$$

where $\rho_{\text {total }}$ is defined by

$$
\rho_{\text {total }}(r):=\rho_{\psi}(r)+\rho_{\phi}(r) .
$$

The charge densities $\rho_{\psi}$ and $\rho_{\phi}$ are given by the variables $u, f$, and $\alpha$ as

$$
\begin{gathered}
\rho_{\psi}=-2 e(e \alpha-\Omega) u^{2}, \\
\rho_{\phi}=-2 e^{2} \alpha f^{2} .
\end{gathered}
$$

We seek configurations of the fields with a nonvanishing value of $\Omega$ that characterizes the solutions.

We require boundary conditions for the fields so that the fields should be regular at the origin. Then, we impose the conditions for the spherically symmetric fields as

$$
\frac{d u}{d r} \rightarrow 0, \quad \frac{d f}{d r} \rightarrow 0, \quad \frac{d \alpha}{d r} \rightarrow 0 \quad \text { as } \quad r \rightarrow 0 .
$$

On the other hand, fields should be in the vacuum state at the spatial infinity. Therefore, from (13), we impose the conditions

$$
u \rightarrow 0, \quad f \rightarrow \eta, \quad \alpha \rightarrow 0 \quad \text { as } r \rightarrow \infty .
$$

\section{NUMERICAL CALCULATIONS}

In this section, we present numerical solutions of the $\mathrm{Q}$ ball by using the relaxation method. In numerics, hereafter, we set $\eta$ as the unit and scale the radial coordinate $r$ as $r \rightarrow \eta r$ and scale the functions $f, u, \alpha$ as $f \rightarrow \eta^{-1} f$, $u \rightarrow \eta^{-1} u, \alpha \rightarrow \eta^{-1} \alpha$, respectively, and scale the parameter $\Omega$ as $\Omega \rightarrow \eta^{-1} \Omega$. We set $\lambda=1, \mu=1.4$ and $e=1$, as an example, in this paper.

In Fig. 1, we plot $u(r), f(r)$, and $\alpha(r)$ as functions of $r$ with four values of $\Omega$. In the all cases of $\Omega$, the functions, whose shapes depend on $\Omega$, have finite support, namely, solitary solutions are obtained.

In the case of $\Omega=1.183$ and $\Omega=1.178$, the field profiles are Gaussian function like. On the other hand, for $\Omega=1.171, \Omega=1.170$, the field profiles are step function like. The solutions in the latter cases represent homogeneous balls, namely, the functions $u, f$, and $\alpha$ take constant values inside the ball, and they change the values quickly in a thin region of the ball surface, $r=r_{s}$, and $u, \alpha$ vanish, and $f$ takes the vacuum expectation value $\eta$ outside the ball.

By numerical calculations, we depict the charge densities $\rho_{\psi}(r)$ and $\rho_{\phi}(r)$ in Fig. 2 as functions of $r$. We find that the charge density $\rho_{\psi}$ is compensated by the counter charge density $\rho_{\phi}$. Then, $\rho_{\text {total }}$ almost vanishes everywhere, namely, perfect screening occurs [20].

As the parameter $\Omega$ varies, the total charge of $\psi, Q_{\psi}$, defined by (9) varies as shown in Fig. 3. The solution exists for $\Omega$ in the range

$$
\Omega_{\min }<\Omega<\Omega_{\max },
$$

where the values of $\Omega_{\min }$ and $\Omega_{\max }$ are discussed later. As seen in Fig. 3, $Q_{\psi}$ diverges at $\Omega=\Omega_{\min }$ and $\Omega=\Omega_{\max }$. For $\Omega$ near $\Omega_{\min }$ in the range (31), the solutions represent homogeneous balls, where the radius of the ball increases as $\Omega$ approaches to $\Omega_{\min }$, while the constant values of $u, f$, and $\alpha$ are independent of $\Omega$. 

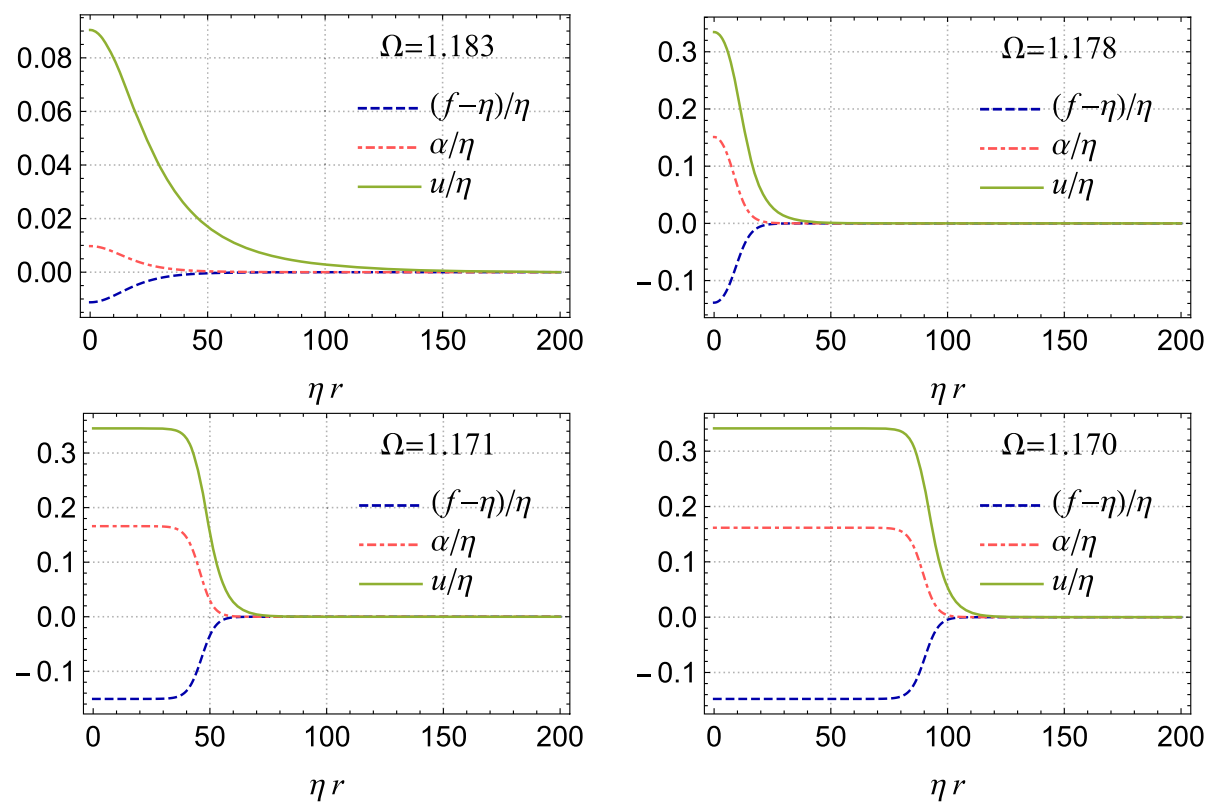

FIG. 1. Numerical solutions $f(r), u(r)$, and $\alpha(r)$ are drawn for $\Omega=1.183,1.178,1.171$, and 1.170.

Here, we estimate the value of $\Omega_{\max }$. Since $u$ is small at a large distance, and $f-\eta$ and $\alpha$ are smaller than $u$ there (see Fig. 1), then solving the linearized equations of (23), we have

$$
u(r) \propto \frac{1}{r} \exp \left(-\sqrt{m_{\psi}^{2}-\Omega^{2}} r\right) .
$$

If we require the solutions are localized in a finite region, the parameter $\Omega$ should satisfy

$$
\Omega^{2}<\Omega_{\max }^{2}:=m_{\psi}^{2}=\mu \eta^{2} .
$$
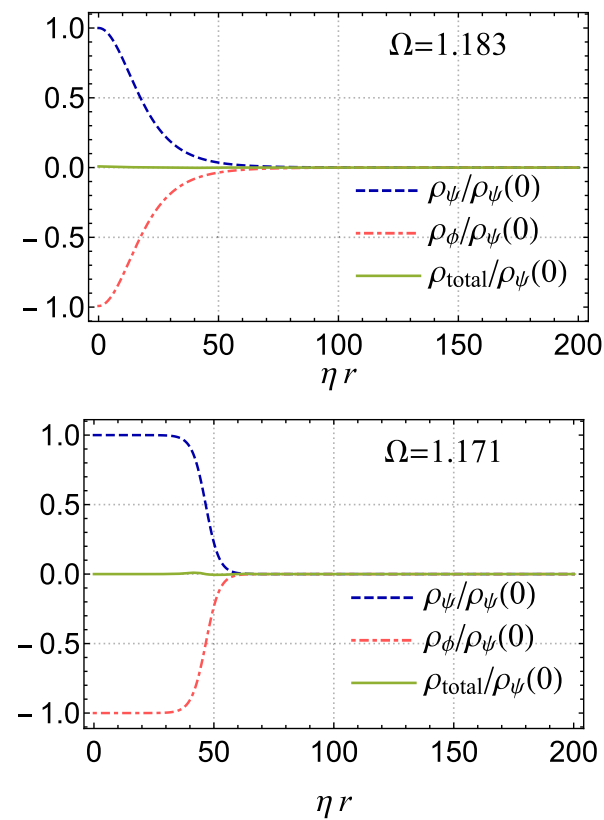

\section{HOMOGENEOUS BALL SOLUTIONS}

For the parameter $\Omega$ very close to $\Omega_{\min }$, the homogeneous ball solutions with a large radius appear. We inspect the homogeneous ball solutions in detail.

The set of Eqs. (23), (24), and (25) can be derived from the effective action in the form,

$$
S_{\mathrm{eff}}=\int r^{2} d r\left(\left(\frac{d u}{d r}\right)^{2}+\left(\frac{d f}{d r}\right)^{2}-\frac{1}{2}\left(\frac{d \alpha}{d r}\right)^{2}-U_{\mathrm{eff}}(u, f, \alpha)\right),
$$
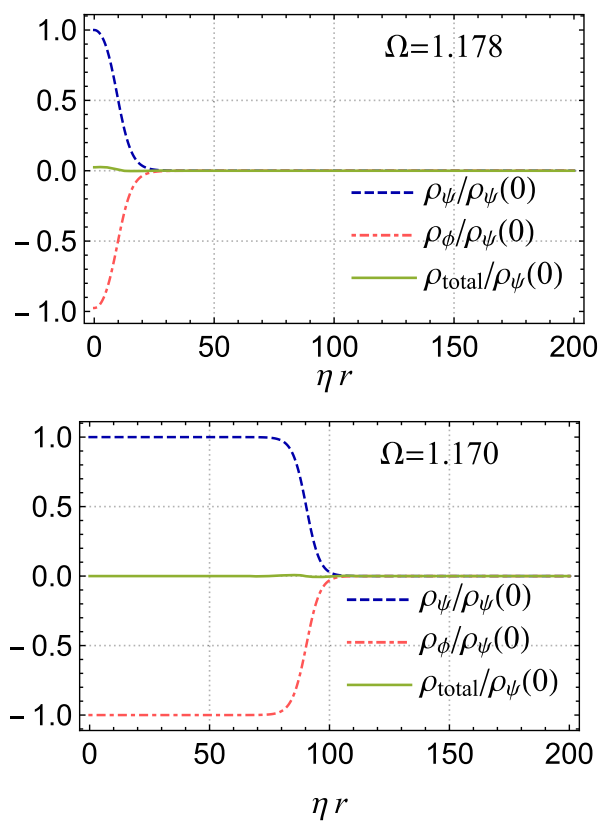

FIG. 2. The charge densities $\rho_{\psi}, \rho_{\phi}$, and $\rho_{\text {total }}:=\rho_{\psi}+\rho_{\phi}$ normalized by the central value of $\rho_{\psi}$ are shown for $\Omega=1.183,1.178,1.171$, and 1.170 . 


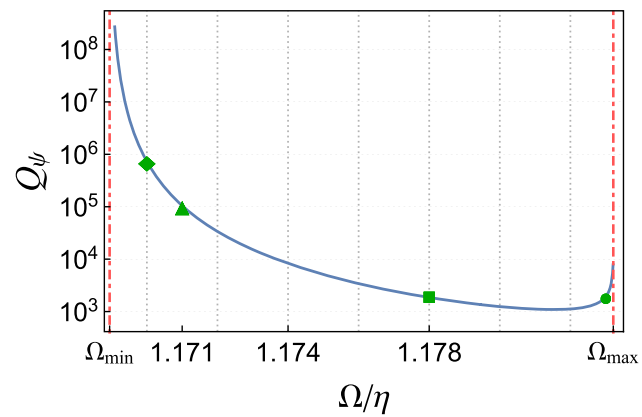

FIG. 3. The total charge of $\psi, Q_{\psi}$, is plotted as a function of $\Omega$. $Q_{\psi}$ diverges at $\Omega=\Omega_{\min }$, dot-dashed line (left), and $\Omega=\Omega_{\max }$, dot-dashed line (right). The value of $\Omega_{\min }$ and $\Omega_{\max }$ are defined by Eqs. (33) and (41), respectively. The circle, square, triangle, and diamond marks in the figure correspond to the cases of $\Omega=1.183,1.178,1.171$, and 1.170 that are shown in Fig. 1 and Fig. 2, respectively.

$U_{\text {eff }}(u, f, \alpha):=-\frac{\lambda}{4}\left(f^{2}-\eta^{2}\right)^{2}-\mu f^{2} u^{2}+e^{2} f^{2} \alpha^{2}+(e \alpha-\Omega)^{2} u^{2}$.

If we regard the coordinate $r$ as a "time", the effective action (34) describes a mechanical system of 3 degrees of freedom (d.o.f.), $u, f$, and $\alpha$, where the "kinetic" term of $\alpha$ has the wrong sign. In the case of the homogeneous ball solution with a large radius, the damping terms that are proportional to $1 / r$ in (23), (24), and (25) are negligible. In this case,

$E_{\mathrm{eff}}:=\left(\frac{d u}{d r}\right)^{2}+\left(\frac{d f}{d r}\right)^{2}-\frac{1}{2}\left(\frac{d \alpha}{d r}\right)^{2}+U_{\mathrm{eff}}(u, f, \alpha)$

is conserved during the motion in the fictitious time $r$.

There are stationary points of the dynamical system on which

$$
\frac{\partial U_{\text {eff }}}{\partial u}=0, \quad \frac{\partial U_{\text {eff }}}{\partial f}=0 \quad \text { and } \quad \frac{\partial U_{\text {eff }}}{\partial \alpha}=0
$$

are satisfied. Two stationary points exist in the region $u \geq 0, f \geq 0$, and $\alpha \geq 0$. One stationary point, say $\mathrm{P}_{\mathrm{v}}$, exists at $(u, f, \alpha)=(0, \eta, 0)$, that is the true vacuum. The other stationary point, say $\mathrm{P}_{0}$, exists at $(u, f, \alpha)=\left(u_{0}, f_{0}, \alpha_{0}\right)$, where $u_{0}, f_{0}$, and $\alpha_{0}$ are given by solving (37) as

$$
\begin{aligned}
& \alpha_{0}=\frac{1}{e(4 \mu-\lambda)}\left((\mu-\lambda) \Omega+\sqrt{\mu(2 \lambda+\mu) \Omega^{2}-\mu \lambda(4 \mu-\lambda) \eta^{2}}\right) \\
& f_{0}=\frac{1}{\sqrt{\mu}}\left(\Omega-e \alpha_{0}\right), \\
& u_{0}=\frac{1}{\sqrt{\mu}} \sqrt{e \alpha_{0}\left(\Omega-e \alpha_{0}\right)} .
\end{aligned}
$$

We note that $0<e \alpha_{0}<\Omega$ should hold for a real value of $u_{0}$. This condition with (33) requires

$$
\lambda<\mu \text {. }
$$

A homogeneous ball solution with a large radius is described by a bounce solution from $\mathrm{P}_{0}$ to $\mathrm{P}_{\mathrm{v}}$. Consider a point in the three-dimensional space $(u, f, \alpha)$ whose motion is governed by the equations of motion (23), (24), and (25). The point that starts in the vicinity of the stationary point $\mathrm{P}_{0}$ spends much "time", $r$, near $\mathrm{P}_{0}$, and traverses to the stationary point $\mathrm{P}_{\mathrm{v}}$ in a short period, and finally stays on $\mathrm{P}_{\mathrm{v}}$. In Fig. 4, the homogeneous ball solution for $\Omega=1.170$ is shown as a trajectory in the $(u, f, \alpha)$ space.

If $\Omega$ approaches to $\Omega_{\min }$, the radius of the homogeneous ball diverges. It means that the solution with an infinitely large radius starts from $\mathrm{P}_{0}$. Since $E_{\text {eff }}$ is conserved for the homogeneous ball solution with a large radius, the bounce solution that describes the homogeneous ball connects the two stationary points with equal potential heights; i.e.,

$$
U_{\text {eff }}\left(\mathrm{P}_{\mathrm{v}}\right)=U_{\text {eff }}\left(\mathrm{P}_{0}\right) .
$$

We see that this occurs for

$\Omega=\Omega_{\min }:=\sqrt{2 \sqrt{\lambda \mu}-\lambda \eta}=\sqrt{m_{\phi}\left(2 m_{\psi}-m_{\phi}\right)}$.

Then, for the parameters satisfying (39), we see

$$
\Omega_{\min }<\Omega_{\max } .
$$

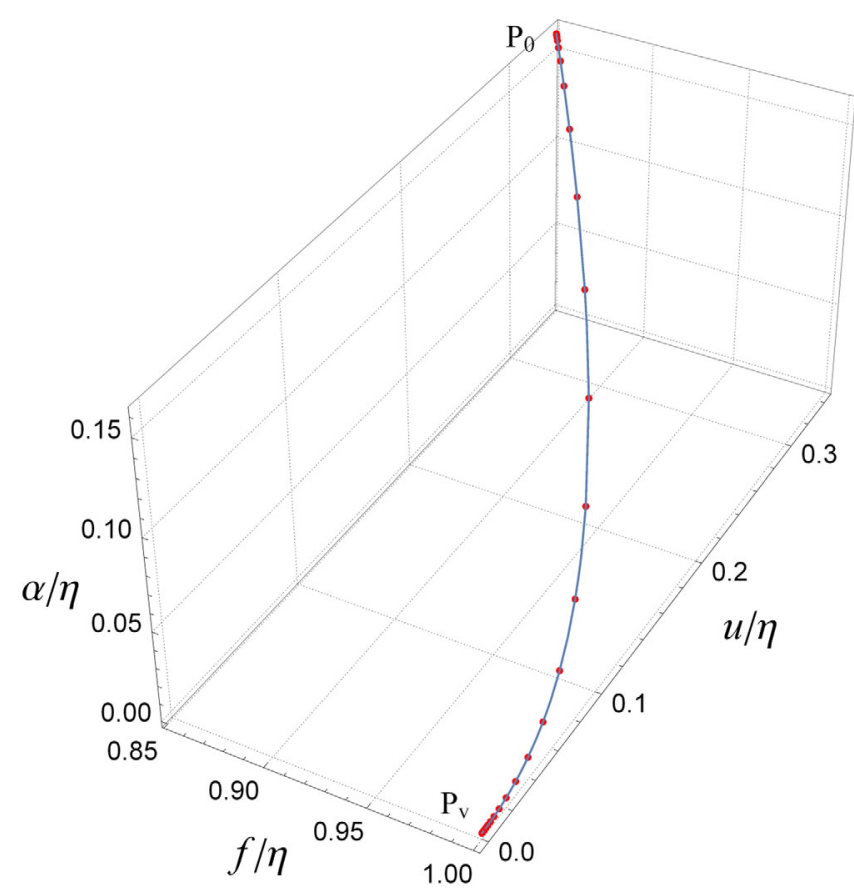

FIG. 4. Trajectory of the numerical solution for $\Omega=1.170$ in the $(u, f, \alpha)$ space. It starts from a point in a vicinity of $\mathrm{P}_{0}$ and ends at $\mathrm{P}_{\mathrm{v}}$. Dots on the trajectory denote laps of the fictitious time $r$. 

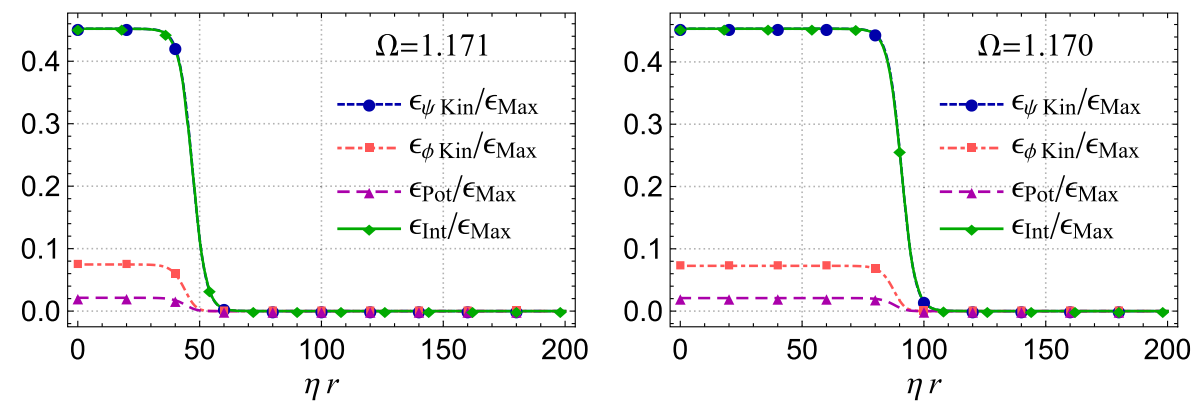

FIG. 5. Components of energy densities of the homogeneous balls normalized by the central value of total energy density are drawn for $\Omega=1.171$ (left panel) and for $\Omega=1.170$ (right panel). The profiles of $\epsilon_{\psi \text { Kin }}$ and $\epsilon_{\text {Int }}$ overlap each other.

Then, the nontopological soliton solutions exist for the model parameters with (39).

We can estimate the value of $\Omega_{\max }$ and $\Omega_{\min }$ given by (33) and (41) using the parameters $\lambda, \mu$, and $\eta$ in the numerical calculations as $\Omega_{\max } \sim 1.1832$ and $\Omega_{\min } \sim 1.1689$. We see in Fig. 3 that numerical calculations reproduce these values.

Using the ansatz (20), (21), and (22), we rewrite the energy (11) for the symmetric system as

$$
\begin{gathered}
E_{\mathrm{NTS}}=4 \pi \int_{0}^{\infty} r^{2} \epsilon(r) d r, \\
\epsilon:=\epsilon_{\psi \text { Kin }}+\epsilon_{\phi \text { Kin }}+\epsilon_{\psi \text { Elast }}+\epsilon_{\phi \text { Elast }}+\epsilon_{\mathrm{Int}}+\epsilon_{\mathrm{Pot}}+\epsilon_{\mathrm{ES}},
\end{gathered}
$$

where

$$
\begin{aligned}
\epsilon_{\psi \text { Kin }}:=\left|D_{t} \psi\right|^{2} & =(e \alpha-\Omega)^{2} u^{2}, \\
\epsilon_{\phi \mathrm{Kin}}:=\left|D_{t} \phi\right|^{2} & =e^{2} f^{2} \alpha^{2}, \\
\epsilon_{\psi \text { Elast }}:=\left(D_{i} \psi\right)^{*}\left(D^{i} \psi\right) & =\left(\frac{d u}{d r}\right)^{2}, \\
\epsilon_{\phi \text { Elast }}:=\left(D_{i} \phi\right)^{*}\left(D^{i} \phi\right) & =\left(\frac{d f}{d r}\right)^{2}, \\
\epsilon_{\mathrm{Pot}}:=V(\phi) & =\frac{\lambda}{4}\left(f^{2}-\eta\right)^{2}, \\
\epsilon_{\mathrm{Int}}:=\mu|\phi|^{2}|\psi|^{2} & =\mu f^{2} u^{2}, \\
\epsilon_{\mathrm{ES}}:=\frac{1}{2} E_{i} E^{i} & =\frac{1}{2}\left(\frac{d \alpha}{d r}\right)^{2}
\end{aligned}
$$

are densities of the kinetic energy of $\psi$ and $\phi$, the elastic energy of $\psi$ and $\phi$, the potential energy of $\phi$, the interaction energy between $\psi$ and $\phi$, and the electrostatic energy, respectively. For the homogeneous ball solutions, these components of energy density are shown in Fig. 5. The dominant components of the energy density $\epsilon$ are $\epsilon_{\psi \operatorname{Kin}}$ and $\epsilon_{\text {Int }}$, and subdominant components are $\epsilon_{\phi \mathrm{Kin}}$ and $\epsilon_{\mathrm{Pot}}$ for the present cases. The densities of the elastic energy and the electrostatic energy, which appear near the surface of the ball, are negligibly small, then, they are not plotted.
We see, from (38), that the dominant and subdominant components of the energy density inside the balls are constants with the values

$$
\begin{aligned}
\epsilon_{\psi \mathrm{Kin}} & =\epsilon_{\mathrm{Int}}=\frac{1}{\mu} e \alpha_{0}\left(\Omega-e \alpha_{0}\right)^{3}, \\
\epsilon_{\phi \mathrm{Kin}} & =\frac{1}{\mu}\left(e \alpha_{0}\right)^{2}\left(\Omega-e \alpha_{0}\right)^{2}, \\
\epsilon_{\mathrm{Pot}} & =\frac{\lambda}{\mu^{2}}\left(\left(\Omega-e \alpha_{0}\right)^{2}-\eta^{2}\right)^{2} .
\end{aligned}
$$

Then the energy density and pressure for the homogeneous ball are constants given by

$$
\begin{aligned}
\epsilon \simeq & \epsilon_{\psi \mathrm{Kin}}+\epsilon_{\phi \mathrm{Kin}}+\epsilon_{\mathrm{Int}}+\epsilon_{\mathrm{Pot}} \\
= & \frac{2}{\mu} e \alpha_{0}\left(\Omega-e \alpha_{0}\right)^{3}+\frac{1}{\mu}\left(e \alpha_{0}\right)^{2}\left(\Omega-e \alpha_{0}\right)^{2} \\
& +\frac{\lambda}{\mu^{2}}\left(\left(\Omega-e \alpha_{0}\right)^{2}-\eta^{2}\right)^{2}, \\
p= & p_{r} \simeq p_{\theta} \\
= & p_{\varphi} \simeq \epsilon_{\psi \mathrm{Kin}}+\epsilon_{\phi \mathrm{Kin}}-\epsilon_{\mathrm{Int}}-\epsilon_{\mathrm{Pot}} \\
= & \frac{1}{\mu}\left(e \alpha_{0}\right)^{2}\left(\Omega-e \alpha_{0}\right)^{2}-\frac{\lambda}{\mu^{2}}\left(\left(\Omega-e \alpha_{0}\right)^{2}-\eta^{2}\right)^{2} .
\end{aligned}
$$

We see that the pressure is almost isotropic and $p \sim 0.05 \mathrm{\epsilon}$ for the homogeneous ball of $\Omega=1.170$. The equation of state of the homogeneous balls is like a nonrelativistic gas.

In the limit $\Omega \rightarrow \Omega_{\min }$, so that $Q_{\psi} \rightarrow \infty$, we see

$$
\begin{aligned}
\epsilon_{\psi \mathrm{Kin}} & =\epsilon_{\mathrm{Int}} \rightarrow \frac{\lambda(\sqrt{\mu}-\sqrt{\lambda}) \sqrt{\mu}}{(2 \sqrt{\mu}-\sqrt{\lambda})^{2}} \eta^{4}, \\
\epsilon_{\phi \mathrm{Kin}} & \rightarrow \lambda\left(\frac{\sqrt{\mu}-\sqrt{\lambda}}{2 \sqrt{\mu}-\sqrt{\lambda}}\right)^{2} \eta^{4}, \\
\epsilon_{\mathrm{Pot}} & \rightarrow \lambda\left(\frac{\sqrt{\mu}-\sqrt{\lambda}}{2 \sqrt{\mu}-\sqrt{\lambda}}\right)^{2} \eta^{4},
\end{aligned}
$$



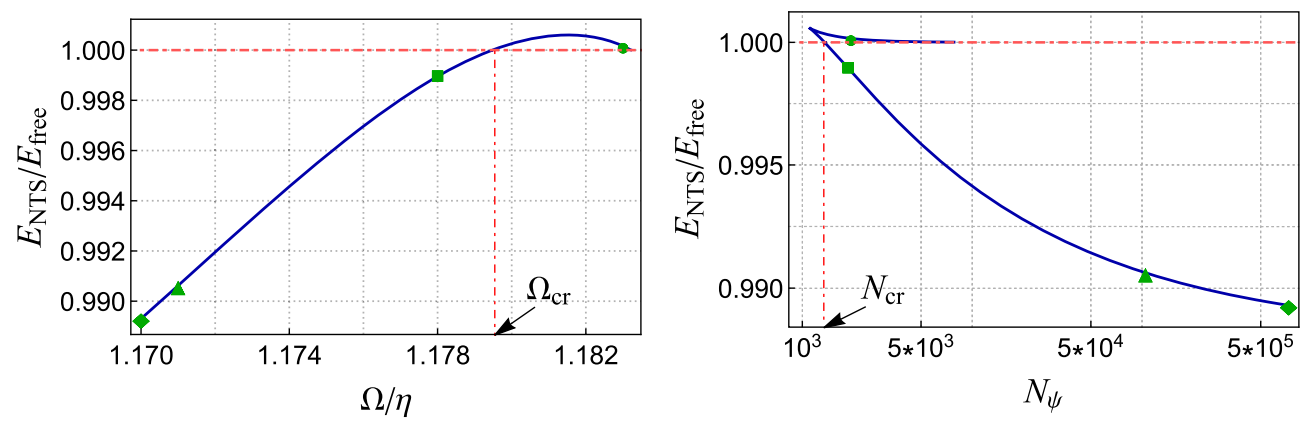

FIG. 6. The energy ratio $E_{\mathrm{NTS}} / E_{\text {free }}$ is plotted as a function of $\Omega$ (left panel), and as a function of $N_{\psi}$ (right panel). The circle, square, triangle, and diamond marks in the figure correspond to the cases of $\Omega=1.183,1.178,1.171$, and 1.170 that are shown in Figs. 1 and 2 , respectively.

then we have

$$
\begin{aligned}
& \epsilon \rightarrow \frac{2 \lambda(\sqrt{\mu}-\sqrt{\lambda})}{2 \sqrt{\mu}-\sqrt{\lambda}} \eta^{4}, \\
& p \rightarrow 0 .
\end{aligned}
$$

Therefore, in the large homogeneous ball limit, the ball becomes a dust ball with a constant energy density given by (49).

\section{STABILITY}

The nontopological soliton, called a Q ball, can be interpreted as a condensate of particles of the scalar field $\psi$, where the Higgs field plays the role of glue against the repulsive force by the $U(1)$ gauge field. We compare the energy of the soliton, $E_{\mathrm{NTS}}$, given by (43) with mass energy of the free particles of $\psi$ that have the same amount of charge of the soliton as a whole. Then, the numbers of the particles are defined by

$$
N_{\psi}:=\frac{Q_{\psi}}{e},
$$

and the mass energy of the free particles of $\psi$ is given by $E_{\text {free }}=m_{\psi} N_{\psi}$.

Figure 6 shows the energy ratio $E_{\mathrm{NTS}} / E_{\text {free }}$ as a function of $\Omega$ and as a function of $N_{\psi}$, respectively. We find a critical value of $\Omega, \Omega_{\mathrm{cr}}$, such that if $\Omega<\Omega_{\mathrm{cr}}, E_{\mathrm{NTS}}<E_{\text {free }}$ holds. Therefore, a $\mathrm{Q}$ ball for $\Omega$ in the range

$$
\Omega_{\min }<\Omega<\Omega_{\mathrm{cr}}
$$

is energetically preferable than the free $\psi$ particles with the same charge of the Q ball as a whole. From the Fig. 6, there exist stable $\mathrm{Q}$ balls that are condensates of large numbers of $\psi$ particles.

Since the energy density and charge density are constant inside the ball, the total energy and the total charge of matter field of the homogeneous ball are written by

$$
E_{\mathrm{NTS}}=\epsilon V, \quad \text { and } \quad Q_{\psi}=\rho_{\psi} V,
$$

where $V$ is the volume of the ball. Then, the energy ratio $E_{\mathrm{NTS}} / E_{\text {free }}$ for the homogeneous ball is calculated as

$$
\frac{E_{\mathrm{NTS}}}{E_{\mathrm{free}}}=\frac{\epsilon V}{m_{\psi} N_{\psi}}=\frac{\epsilon Q_{\psi} / \rho_{\psi}}{m_{\psi} Q_{\psi} / e}=\frac{e \epsilon}{m_{\psi} \rho_{\psi}} .
$$

In the limit $\Omega \rightarrow \Omega_{\min }$, so that $Q_{\psi} \rightarrow \infty$, we obtain $E_{\mathrm{NTS}} / E_{\text {free }}$ as

$$
\frac{E_{\mathrm{NTS}}}{E_{\mathrm{free}}} \rightarrow((2-\sqrt{\lambda / \mu}) \sqrt{\lambda / \mu})^{1 / 2} .
$$

It is clear that $E_{\mathrm{NTS}} / E_{\text {free }}<1$ for $\lambda<\mu$ in the limit $\Omega \rightarrow \Omega_{\min }$. Therefore, the large limit of the homogeneous ball solution is stable.

We show $E_{\mathrm{NTS}} / E_{\text {free }}$ for various $Q_{\psi}$ in Table I. We see the inequality

$$
E_{\mathrm{NTS}}\left(Q_{\psi 1}\right)+E_{\mathrm{NTS}}\left(Q_{\psi 2}\right)>E_{\mathrm{NTS}}\left(Q_{\psi 1}+Q_{\psi 2}\right)
$$

TABLE I. The total charge of $\psi, Q_{\psi}$, and total energy, $E_{\mathrm{NTS}}$, of $\mathrm{Q}$ balls for various values of parameters $\Omega$.

\begin{tabular}{lcc}
\hline \hline$\Omega$ & $Q_{\psi}$ & $E_{\mathrm{NTS}}$ \\
\hline 1.17771 & 2000 & 2363.4 \\
1.17559 & 4000 & 4716.3 \\
1.17465 & 6000 & 7066.4 \\
1.17407 & 8000 & 9415.1 \\
1.17368 & 10000 & 11762.8 \\
1.17262 & 20000 & 23493.5 \\
1.17213 & 30000 & 35217.0 \\
1.17182 & 40000 & 46936.7 \\
1.17161 & 50000 & 58653.7 \\
1.17103 & 100000 & 117217 \\
1.17059 & 200000 & 234295 \\
1.17037 & 300000 & 351342 \\
1.17024 & 400000 & 468373 \\
1.17015 & 500000 & 585392 \\
\hline \hline
\end{tabular}


holds for any $Q_{\psi 1}$ and $Q_{\psi 2}$ in the table. It means that one large $\mathrm{Q}$ ball is energetically preferable to two small $\mathrm{Q}$ balls. Therefore, two Q balls can merge into a $\mathrm{Q}$ ball, but a $\mathrm{Q}$ ball does not decay into two Q balls.

\section{SUMMARY AND DISCUSSIONS}

In this paper, we have studied the coupled system of a complex matter scalar field, a U(1) gauge field, and a complex Higgs scalar field with a potential that causes spontaneous symmetry breaking. This is a generalization of the Friedberg-Lee-Sirlin model [1]. In this system, a local $\mathrm{U}(1) \times$ global $\mathrm{U}(1)$ symmetry is broken spontaneously into a global U(1) symmetry by the Higgs field. We have shown numerically that there are spherically symmetric nontopological soliton solutions, Q balls, that are characterized by a phase rotation of the complex matter scalar field, $\Omega$. The $\mathrm{Q}$ balls can exist for a finite range of $\Omega$, and there are two types of solutions: Gaussian balls and homogeneous balls.

In the homogeneous ball solutions, the fields take constant values inside the ball, and they change the values quickly at the ball surface to the vacuum values outside the ball. The charge density of matter scalar field that arises inside the ball is canceled out everywhere by the counter charge cloud of the Higgs and the gauge fields, namely, perfect screening occurs $[19,20]$. Inspecting the energymomentum tensor of the fields, we have shown that the energy density and pressure inside the balls take constant values. The pressure is almost isotropic, and the value is much less than the energy density. Then, a homogeneous ball is like a ball of homogeneous nonrelativistic gas.

Homogeneous ball solutions appear as "Q matters" in the system of a self-coupling single complex scalar field studied by Coleman [2]. These solutions are interpreted as bounce solutions that connect two stationary points of the potential of 1 d.o.f. In the extended system by introducing a $\mathrm{U}(1)$ gauge field, the homogeneous ball solution does not appear. In the gauged system, since a repulsive force acting between charges pushes them outward to the surface of the ball, then the solution has a radial inhomogeneity [14-16]. In contrast, in the gauged system with the spontaneous symmetry breaking investigated in this paper, the perfect screening of charge occurs, then no repulsive force acts inside the ball. Therefore, the homogeneous ball solutions can exist. This is suggested in the work [21]. The homogeneous ball solutions are interpreted as bounce solutions that connect two stationary points of the potential of 3 d.o.f. Then, the homogeneous ball solutions obtained in this paper are extensions of Coleman's Q matters.

By comparison of the energies, it was shown that if the charge of the matter field is greater than a critical value, a $Q$ ball is stable against dispersion into free particles and against decay into two smaller Q balls. In addition to the analysis in this paper, it is important to investigate the stability in various viewpoints [22-27].
In the extended systems by the gauge field without the Higgs field, the size of a stable charged Q ball has an upper bound [14-16]. In contrast, a stable charge screened homogeneous ball has no limit of mass. Of cause, this is true as far as the gravity can be neglected. If the mass of the homogeneous ball becomes too large so that the pressure fails to sustain the gravity, the ball would collapse to a black hole. Then, there exists an upper bound of mass for the stable homogeneous ball if the gravity is taken into account. It is an interesting issue to study the gravitational effects on the Q balls [28-32]. We would report this issue on the present system in a forthcoming paper.

The Q balls obtained in this paper would have applications in cosmology and astrophysics. The perfect screening of the charge is a preferable property for the gauged Q balls to be dark matter [6-10]. It is an important issue how much amount of the $\mathrm{Q}$ balls are produced in the evolution of the Universe [33-38]. It would be an interesting problem to clarify the mass distribution spectrum of the $\mathrm{Q}$ balls, which would evolve by a merging process of $\mathrm{Q}$ balls, in the present stage of the Universe.

In the model studied in this paper, we assumed that matter is described by a complex scalar field, for simplicity. It is interesting to consider fermionic matter fields that form Q balls. Indeed, fermionic Q balls are already studied [39-42], but a large fermionic soliton is hardly produced because of the Pauli exclusion principle. If two fermions make a bosonic bound state as in a superconductor, it is expected that the charge screened large $\mathrm{Q}$ ball as was discussed in this paper, would be possible. To clarify this possibility would be a challenging work.

\section{ACKNOWLEDGMENTS}

We would like to thank K.-i. Nakao, H. Itoyama, Y. Yasui, N. Maru, N. Sakai, and M. Minamitsuji for valuable discussion. H. I. was supported by JSPS KAKENHI Grant No. 16K05358.

\section{APPENDIX: ENERGY-MOMENTUM TENSOR OF THE SYSTEM}

The energy-momentum tensor $T_{\mu \nu}$ of the present system is given by

$$
\begin{aligned}
T_{\mu \nu}= & 2\left(D_{\mu} \psi\right)^{*}\left(D_{\nu} \psi\right)-g_{\mu \nu}\left(D_{\alpha} \psi\right)^{*}\left(D^{\alpha} \psi\right) \\
& +2\left(D_{\mu} \phi\right)^{*}\left(D_{\nu} \phi\right)-g_{\mu \nu}\left(D_{\alpha} \phi\right)^{*}\left(D^{\alpha} \phi\right) \\
& -g_{\mu \nu}\left(V(\phi)+\mu \psi^{*} \psi \phi^{*} \phi\right) \\
& +\left(F_{\mu \alpha} F_{\nu}{ }^{\alpha}-\frac{1}{4} g_{\mu \nu} F_{\alpha \beta} F^{\alpha \beta}\right) .
\end{aligned}
$$

Energy density and pressure components are given by 


$$
\begin{aligned}
\epsilon=-T_{t}^{t}= & \left|D_{t} \psi\right|^{2}+\left(D_{i} \psi\right)^{*}\left(D^{i} \psi\right)+\left|D_{t} \phi\right|^{2}+\left(D_{i} \phi\right)^{*}\left(D^{i} \phi\right)+V(\phi)+\mu|\psi|^{2}|\phi|^{2}+\frac{1}{2}\left(E_{i} E^{i}+B_{i} B^{i}\right), \\
p_{r}=T_{r}^{r}= & \left(D_{r} \psi\right)^{*}\left(D^{r} \psi\right)+\left|D_{t} \psi\right|^{2}-\left(D_{\theta} \psi\right)^{*}\left(D^{\theta} \psi\right)-\left(D_{\varphi} \psi\right)^{*}\left(D^{\varphi} \psi\right)+\left(D_{r} \phi\right)^{*}\left(D^{r} \phi\right)+\left|D_{t} \phi\right|^{2}-\left(D_{\theta} \phi\right)^{*}\left(D^{\theta} \phi\right) \\
& -\left(D_{\varphi} \phi\right)^{*}\left(D^{\varphi} \phi\right)-V(\phi)-\mu|\psi|^{2}|\phi|^{2}+\frac{1}{2}\left(-E_{r} E^{r}+E_{\theta} E^{\theta}+E_{\varphi} E^{\varphi}-B_{r} B^{r}+B_{\theta} B^{\theta}+B_{\varphi} B^{\varphi}\right), \\
p_{\theta}=T_{\theta}^{\theta}= & \left(D_{\theta} \psi\right)^{*}\left(D^{\theta} \psi\right)+\left|D_{t} \psi\right|^{2}-\left(D_{r} \psi\right)^{*}\left(D^{r} \psi\right)-\left(D_{\varphi} \psi\right)^{*}\left(D^{\varphi} \psi\right)+\left(D_{\theta} \phi\right)^{*}\left(D^{\theta} \phi\right)+\left|D_{t} \phi\right|^{2}-\left(D_{r} \phi\right)^{*}\left(D^{r} \phi\right) \\
& -\left(D_{\varphi} \phi\right)^{*}\left(D^{\varphi} \phi\right)-V(\phi)-\mu|\psi|^{2}|\phi|^{2}+\frac{1}{2}\left(-E_{\theta} E^{\theta}+E_{r} E^{r}+E_{\varphi} E^{\varphi}-B_{\theta} B^{\theta}+B_{r} B^{r}+B_{\varphi} B^{\varphi}\right), \\
p_{\varphi}=T_{\varphi}^{\varphi}= & \left(D_{\varphi} \psi\right)^{*}\left(D^{\varphi} \psi\right)+\left|D_{t} \psi\right|^{2}-\left(D_{r} \psi\right)^{*}\left(D^{r} \psi\right)-\left(D_{\theta} \psi\right)^{*}\left(D^{\theta} \psi\right)+\left(D_{\varphi} \phi\right)^{*}\left(D^{\varphi} \phi\right)+\left|D_{t} \phi\right|^{2}-\left(D_{r} \phi\right)^{*}\left(D^{r} \phi\right) \\
& -\left(D_{\theta} \phi\right)^{*}\left(D^{\theta} \phi\right)-V(\phi)-\mu|\psi|^{2}|\phi|^{2}+\frac{1}{2}\left(-E_{\varphi} E^{\varphi}+E_{r} E^{r}+E_{\theta} E^{\theta}-B_{\varphi} B^{\varphi}+B_{r} B^{r}+B_{\theta} B^{\theta}\right) .
\end{aligned}
$$

[1] R. Friedberg, T. D. Lee, and A. Sirlin, Phys. Rev. D 13, 2739 (1976).

[2] S. R. Coleman, Nucl. Phys. B262, 263 (1985); B269, 744 (E) (1986).

[3] A. Kusenko, Phys. Lett. B 405, 108 (1997).

[4] G. R. Dvali, A. Kusenko, and M. E. Shaposhnikov, Phys. Lett. B 417, 99 (1998).

[5] S. Kasuya and M. Kawasaki, Phys. Rev. Lett. 85, 2677 (2000).

[6] A. Kusenko and M. E. Shaposhnikov, Phys. Lett. B 418, 46 (1998).

[7] A. Kusenko and P. J. Steinhardt, Phys. Rev. Lett. 87, 141301 (2001).

[8] M. Fujii and K. Hamaguchi, Phys. Lett. B 525, 143 (2002).

[9] K. Enqvist, A. Jokinen, T. Multamaki, and I. Vilja, Phys. Lett. B 526, 9 (2002).

[10] A. Kusenko, L. Loveridge, and M. Shaposhnikov, Phys. Rev. D 72, 025015 (2005).

[11] K. Enqvist and J. McDonald, Phys. Lett. B 425, 309 (1998).

[12] S. Kasuya and M. Kawasaki, Phys. Rev. D 61, 041301 (2000).

[13] M. Kawasaki, F. Takahashi, and M. Yamaguchi, Phys. Rev. D 66, 043516 (2002).

[14] K. M. Lee, J. A. Stein-Schabes, R. Watkins, and L. M. Widrow, Phys. Rev. D 39, 1665 (1989).

[15] X. Shi and X. Z. Li, J. Phys. A 24, 4075 (1991).

[16] I. E. Gulamov, E. Y. Nugaev, A. G. Panin, and M. N. Smolyakov, Phys. Rev. D 92, 045011 (2015).

[17] H. Arodz and J. Lis, Phys. Rev. D 79, 045002 (2009).

[18] T. Tamaki and N. Sakai, Phys. Rev. D 90, 085022 (2014).

[19] H. Ishihara and T. Ogawa, Prog. Theor. Exp. Phys. 2019, 021B01 (2019).

[20] H. Ishihara and T. Ogawa, arXiv:1811.10848.
[21] K. N. Anagnostopoulos, M. Axenides, E. G. Floratos, and N. Tetradis, Phys. Rev. D 64, 125006 (2001).

[22] A. G. Cohen, S. R. Coleman, H. Georgi, and A. Manohar, Nucl. Phys. B272, 301 (1986).

[23] A. Kusenko, Phys. Lett. B 404, 285 (1997).

[24] T. Multamaki and I. Vilja, Nucl. Phys. B574, 130 (2000).

[25] F. P. Correia and M. G. Schmidt, Eur. Phys. J. C 21, 181 (2001).

[26] M. Kawasaki, K. Konya, and F. Takahashi, Phys. Lett. B 619, 233 (2005).

[27] N. Sakai and M. Sasaki, Prog. Theor. Phys. 119, 929 (2008).

[28] R. Friedberg, T. D. Lee, and Y. Pang, Phys. Rev. D 35, 3640 (1987).

[29] R. Friedberg, T. D. Lee, and Y. Pang, Phys. Rev. D 35, 3658 (1987).

[30] T. D. Lee and Y. Pang, Phys. Rev. D 35, 3678 (1987).

[31] B. W. Lynn, Nucl. Phys. B321, 465 (1989).

[32] E. W. Mielke and F. E. Schunck, Phys. Rev. D 66, 023503 (2002).

[33] J. A. Frieman, G. B. Gelmini, M. Gleiser, and E. W. Kolb, Phys. Rev. Lett. 60, 2101 (1988).

[34] K. Griest and E. W. Kolb, Phys. Rev. D 40, 3231 (1989).

[35] S. Kasuya and M. Kawasaki, Phys. Rev. D 62, 023512 (2000).

[36] M. Postma, Phys. Rev. D 65, 085035 (2002).

[37] T. Multamaki and I. Vilja, Phys. Lett. B 535, 170 (2002).

[38] T. Hiramatsu, M. Kawasaki, and F. Takahashi, J. Cosmol. Astropart. Phys. 06 (2010) 008.

[39] R. Friedberg and T. D. Lee, Phys. Rev. D 15, 1694 (1977).

[40] R. Friedberg and T. D. Lee, Phys. Rev. D 16, 1096 (1977).

[41] K. Shima, Nuovo Cimento A 44, 163 (1978).

[42] T. S. Levi and M. Gleiser, Phys. Rev. D 66, 087701 (2002). 